\title{
The flavor-blind principle: A symmetrical approach to the Gatto-Sartori-Tonin relation
}

\author{
U. J. Saldaña-Salazar* \\ Departamento de Física, Centro de Investigación y de Estudios Avanzados del IPN, \\ Apdo. Postal 14-740, 07000, México D.F., México.
}

August 2, 2018

\begin{abstract}
We perform a systematic study of the generic Gatto-Sartori-Tonin relation, $\tan ^{2} \theta_{i j}=m_{i} / m_{j}$. This study of fermion mixing phenomena leads us to the necessary conditions that are needed in order to obtain it without any approximation. We begin by considering two Dirac fermion families. By means of the hierarchy in the masses, it is found that a sufficient and necessary condition is to have a normal matrix with $m_{11}=0$. This matrix can be decomposed into two different linearly independent contributions. The origin for such two independent contributions can be naturally explained by what we shall call the flavor-blind principle. This principle states that Yukawa couplings shall be either flavor-blind or decomposed into several sets obeying distinct permutation symmetries. In general, it is shown that the symmetry properties of the introduced set of Yukawa matrices follow for $n$ fermion families the unique sequential breaking $S_{n L} \otimes S_{n R} \rightarrow$ $S_{(n-1) L} \otimes S_{(n-1) R} \rightarrow \cdots \rightarrow S_{2 L} \otimes S_{2 R} \rightarrow S_{2 A}$. The particular case of three fermion families explains why the four mass ratios parametrization that we recently proposed can be used even in the case of no hierarchical masses.
\end{abstract}

\section{Introduction}

Family mixing in both the quark and lepton sectors is nowadays a well established fact. It provides us with a description on how flavor transitions are produced when fermions interact with the charged

${ }^{*}$ E-mail: ulisesjesus@protonmail.ch 
bosons and also, in the case of neutrinos, through their propagation. The rate of these transitions are measured by the moduli of the mixing matrix elements whereas the amount of Charge-Parity $(\mathrm{CP})$ violation by the corresponding Jarlskog invariant. The Standard Model of the electroweak interactions plus massive neutrinos, successfully describes this picture with ten physical parameters per sector (in the case of Majorana neutrinos, two more phases). Nevertheless, since the late sixties of the past century, the possibility of having relations among the mixing angles and fermion mass ratios was realized. The pioneering work of Gatto, Sartori, and Tonin showed a relation satisfying, with good accuracy, the experimental value of the Cabibbo angle [1]

$$
\tan ^{2} \theta_{C} \approx \frac{m_{d}}{m_{s}}
$$

At present, a more complete relation with greater accuracy includes the contribution coming from the up-quark sector and a small correction through the denominator [2, 3]

$$
\tan ^{2} \theta_{C} \approx \frac{\hat{m}_{d}+\hat{m}_{u}}{\left(1+\hat{m}_{d}\right)\left(1+\hat{m}_{u}\right)},
$$

with $\hat{m}_{d}=m_{d} / m_{s}$ and $\hat{m}_{u}=m_{u} / m_{c}$. From now on, we will refer to it, in its generic form which only encompasses a single fermion species, $\tan ^{2} \theta_{i j}=m_{i} / m_{j}$, as the Gatto-Sartori-Tonin (GST) relation, for a review on this topic please refer to [4]. It has been already recognized by different authors that not only the observed hierarchy in the quark masses [3-13]

$$
\begin{aligned}
& m_{t}\left(M_{Z}\right) \gg m_{c}\left(M_{Z}\right) \gg m_{u}\left(M_{Z}\right), \\
& m_{b}\left(M_{Z}\right) \gg m_{s}\left(M_{Z}\right) \gg m_{d}\left(M_{Z}\right),
\end{aligned}
$$

can give access to desired GST relations, but also the charged leptons hierarchy plus the possible milder one in the neutrino masses [3, 13, 15]

$$
\begin{gathered}
m_{\tau}\left(M_{Z}\right) \gg m_{\mu}\left(M_{Z}\right) \gg m_{e}\left(M_{Z}\right), \\
m_{\nu 3}^{2}\left(M_{Z}\right) \gg m_{\nu 2}^{2}\left(M_{Z}\right) \gg m_{\nu 1}^{2}\left(M_{Z}\right) .
\end{gathered}
$$

However, only until recently, a procedure to build a mixing parametrization in terms of only the four independent mass ratios of the corresponding fermion sector was fully achieved [3]. This new parametrization makes one to wonder about the possible physical meaning of the GST relation and the necessary conditions to find it in an exact way. Moreover, the procedure allowed the computation of the neutrino masses predicting the mass values [3]

$$
\begin{aligned}
& m_{\nu 1}=(0.0041 \pm 0.0015) \mathrm{eV} \\
& m_{\nu 2}=(0.0096 \pm 0.0005) \mathrm{eV} \\
& m_{\nu 3}=(0.050 \pm 0.001) \mathrm{eV}
\end{aligned}
$$


From them, the study of the leptonic mixing was made possible and was found to be in excellent agreement to the most recent global fits [3]. Nevertheless, the existing mild hierarchy in the neutrino masses cannot wholly justify this procedure which was obtained from the strong hierarchy in the quark masses. Therefore, the only reason to explain such an excellent agreement in the leptonic mixing despite the mild hierarchy in the neutrino masses is to disentangle the origin of the hierarchical nature from the dynamics responsible for fermion mixing, see for example [16]. In the following, we leave aside the issue of hierarchical masses and only focus on what is being responsible for fermion mixing.

Yukawa interactions between the different fermion families with the Higgs field are only requested to be renormalizable and gauge invariant. As a consequence, Yukawa couplings appear as a set of arbitrary complex parameters. But, could this set of Yukawa couplings follow some principle? In this work we try to answer this question. We take the study of the GST relation in the two family case as our starting point. We find that a Yukawa matrix should be normal with its first diagonal element equal to zero, $y_{11}=0$, to be necessary and sufficient in order to be diagonalized by the GST relation. This in return permits us to distinguish two different hierarchical structures, $y_{22} \gg y_{i j}$; their symmetric or antisymmetric properties thus pointing out to a possible symmetrical origin. This basis is commonly known as heavy or hierarchical basis, as suggested by its structure. Nonetheless, within this initial basis, we note that the dominant contribution is invariant under a $U(1)^{3}$ global symmetry whereas the small contribution breaks it. An appropriate weak basis transformation can put them into equal footing.1 In the new basis, it may be read (what we shall call the flavor-blind principle): Yukawa couplings shall be either flavor-blind or decomposed into several sets obeying distinct permutation symmetries. This statement for the three family case was first initiated but partially by Harari, Haut, and Weyers [17] and later reconsidered with different approaches by other authors [2, 18,-26]. The main idea was to consider, as onset, the so called "democratic form" which is invariant under permutations of the columns and/or rows, and described by the symmetric group $S_{3 L} \otimes S_{3 R}$. Afterwards, the addition of further terms would explicitly break it into various ways. Here we show that the flavor-blind principle gives, on its own, all the necessary information to build up the Yukawa matrices; putting aside, the initial arbitrariness with which they were introduced. Our approach differs from previous ones in several aspects:2

1. We assume as our starting point that fermion mixing is described by the GST relation.

2. From it, we find two necessary and sufficient mathematical conditions to be satisfied by a Yukawa matrix: normality and its $1-1$ element equal to zero.

3. This mathematical condition can be reexpressed as a physical statement, which we call the

\footnotetext{
${ }^{1} \mathrm{~A}$ weak basis transformation is such that the charged current remains diagonal after it.

${ }^{2}$ Recall that we do not intend here to give an explanation to the hierarchical nature of fermion masses, but rather show how mixing phenomena are driven by the flavor-blind principle. For example, the formalism to be developed here could complement recent works treating the hierarchy in the masses [16, 27,-29].
} 
flavor-blind principle: Yukawa couplings shall be either flavor-blind or decomposed into several sets obeying distinct permutation symmetries.

4. The main purpose pursued in earlier works [2,17,-26] was to build mass matrices with some of its elements being equal to zero, and therefore justify how texture zeros arise. The symmetry was explicitly broken from $S_{3 L} \otimes S_{3 R}$ to either $S_{2 L} \otimes S_{2 R}$ [2,20,22,24-26] or completely [1719, 21, 23]. In the former case, it would afterwards be broken completely by terms either being $S_{3}^{\text {diag }}$ symmetrical [2, 20, 25, 26] or without any symmetry at all [22, 24]. These different ways seem to follow only a subjective choice. Here we want to stress that this is not the case and the breaking pattern follows a unique ordering

$$
S_{3 L} \otimes S_{3 R} \rightarrow S_{2 L} \otimes S_{2 R} \rightarrow S_{2 A}
$$

5. The latter symmetry breaking pattern is partially equivalent to the minimal breaking of the flavor symmetry group which appears as an accidental global symmetry when Yukawa interactions are turned off

$$
U(3)^{6} \rightarrow U(2)^{6} \rightarrow U(1)^{6} \rightarrow U(1)_{B} \otimes U(1)_{L},
$$

but it is not completely equivalent as it lacks an explanation to the hierarchical nature of fermion masses.

6. In general, the flavor-blind principle applied to the $n$ family case implies the symmetry breaking pattern

$$
S_{n L} \otimes S_{n R} \rightarrow S_{(n-1) L} \otimes S_{(n-1) R} \rightarrow \cdots \rightarrow S_{3 L} \otimes S_{3 R} \rightarrow S_{2 L} \otimes S_{2 R} \rightarrow S_{2 A} .
$$

This paper is organized in the following way. In Section 2, we study the two fermion family case. We begin by finding the necessary conditions a mass matrix should satisfy to be diagonalized by the GST relation. Afterwards, we study the implications of demanding one of the mass matrix elements to be equal to zero. In Section 3, we find a symmetrical origin which not only works in the two family case but also for the three family one. Finally, we conclude. In the Appendix A we remark the universal nature of the GST relation by decoupling two quantum harmonic oscillators with equal frequencies but different masses; while in Appendix B we apply the flavor-blind principle to the $n$ family case and show why the last step of the symmetry breaking pattern is $S_{2 A}$ and not $S_{2 S}$.

\section{Two fermion families}

The GST relation is a universal relation. By this we mean that it appears in different kinds of physical phenomena, as for example, when decoupling two coupled quantum harmonic oscillators with different masses but equal frequencies, see Appendix A. In general, the relation may be derived from the 
quadratic equation

$$
\tan ^{2} \theta+\frac{m_{2}-m_{1}}{\sqrt{m_{1} m_{2}}} \tan \theta-1=0
$$

which has two solutions:

$$
\tan \theta=\left\{\begin{array}{l}
-\sqrt{\frac{m_{2}}{m_{1}}} \\
+\sqrt{\frac{m_{1}}{m_{2}}}
\end{array},\right.
$$

corresponding the latter to the GST relation.

What are the necessary and sufficient conditions to obtain this relation in an exact manner within the context of two fermion families mixing?

\subsection{Finding out the conditions}

Following a similar procedure as the authors in [3] we can use the hierarchy in the masses to study the matrix elements. The implied most general form for the mass matrices is a normal matrix given as 3

$$
\mathbf{m}=\left(\begin{array}{cc}
\kappa\left|m_{12}\right|^{2} e^{i \delta_{11}} & \left|m_{12}\right| e^{i \delta_{12}} \\
\left|m_{12}\right| e^{i \delta_{21}} & \left|m_{22}\right| e^{i \delta_{22}}
\end{array}\right),
$$

where the coefficient $\kappa$ has been introduced in order to track the contributions coming from $m_{11}$.

The obtention of the unitary transformation which enters in the charged current interactions requires the study of the left Hermitian product, $\mathbf{m m}^{\dagger}$. In order to put this product into diagonal form we require, in general, a unitary transformation with the rotation angle satisfying

$$
\tan ^{2} \theta-\left(\frac{n_{11}-n_{22}}{\left|n_{12}\right|}\right) \tan \theta-1=0,
$$

where we have denoted the left Hermitian product by $\mathbf{n}=\mathbf{m m}^{\dagger}$ and its matrix elements are

$$
\begin{gathered}
n_{11}=\kappa^{2}\left|m_{12}\right|^{4}+\left|m_{12}\right|^{2}, \quad n_{22}=\left|m_{12}\right|^{2}+\left|m_{22}\right|^{2}, \\
n_{12}=\kappa\left|m_{12}\right|^{3} e^{i\left(\delta_{11}-\delta_{21}\right)}+\left|m_{12}\right|\left|m_{22}\right| e^{i\left(\delta_{12}-\delta_{22}\right)} .
\end{gathered}
$$

\footnotetext{
${ }^{3}$ The contribution of $\mathbf{m}_{11}$ in $\mathbf{m m}^{\dagger}$, if we assume it to be much smaller than one, could be neglected in the calculations [3]. However, we will leave out any aproximations and stick to the general case.
} 
On the other hand, if what we want is to reproduce the GST relation, then Eqs. (10) and (13) imply our first condition which is sufficient to produce the desired relation

$$
\frac{n_{11}-n_{22}}{\left|n_{12}\right|}=\frac{m_{1}-m_{2}}{\sqrt{m_{1} m_{2}}}
$$

In terms of matrix elements it reads

$$
\frac{\kappa^{2}\left|m_{12}\right|^{4}-\left|m_{22}\right|^{2}}{\sqrt{\kappa^{2}\left|m_{12}\right|^{6}+\left|m_{12}\right|^{2}\left|m_{22}\right|^{2}+2 \kappa\left|m_{12}\right|^{4}\left|m_{22}\right| \cos \left(\delta_{11}+\delta_{22}-\left(\delta_{12}+\delta_{21}\right)\right)}}=\frac{m_{1}-m_{2}}{\sqrt{m_{1} m_{2}}} .
$$

At this point, it is instructive to make use of the two invariants of the mass matrix

$$
\begin{array}{r}
\operatorname{Tr}(\mathbf{n})=n_{11}+n_{22}=m_{1}^{2}+m_{2}^{2}, \\
\operatorname{det}(\mathbf{n})=n_{11} n_{22}-\left|n_{12}\right|^{2}=m_{1}^{2} m_{2}^{2},
\end{array}
$$

which in terms of the matrix elements are

$$
\begin{array}{r}
\kappa^{2}\left|m_{12}\right|^{4}+2\left|m_{12}\right|^{2}+\left|m_{22}\right|^{2}=m_{1}^{2}+m_{2}^{2}, \\
\left|m_{12}\right|^{4}\left[\kappa^{2}\left|m_{22}\right|^{2}-2 \kappa\left|m_{22}\right| \cos \left(\delta_{11}+\delta_{22}-\left(\delta_{12}+\delta_{21}\right)\right)+1\right]=m_{1}^{2} m_{2}^{2} .
\end{array}
$$

A first thing to notice here is that we have an undesired dependence on a linear combination of phases. Recall that the GST relation only depends on the masses and we want to have this same dependence on the moduli of the mass matrix elements, $\left|m_{i j}\right|=\left|m_{i j}\right|\left(m_{1}, m_{2}\right) 4$ This in return will give us Yukawa couplings whose magnitude will only depend on the masses. To get rid off this kind of phase dependence then we require to either have $m_{11}$ or $m_{22}$ equal to zero. In fact, the only viable option consistent with the hierarchical structure of $\mathbf{m m}^{\dagger}$ is $m_{11}=0$. So we set $\kappa=0$ and obtain a solution consistent with Eq. (16),

$$
\left|m_{12}\right|=\sqrt{m_{1} m_{2}}, \quad \quad\left|m_{22}\right|=m_{2}-m_{1} .
$$

We then conclude that setting $\kappa=0$ is necessary if we want the magnitude of the Yukawa couplings to only depend on the masses and not on phases coming from the complex matrix elements.

Hence, we find the form of a complex matrix sufficient to reproduce the GST relation by

$$
\mathbf{m}=\left(\begin{array}{cc}
0 & \sqrt{m_{1} m_{2}} e^{i \delta_{12}} \\
\sqrt{m_{1} m_{2}} e^{i \delta_{21}} & \left(m_{2}-m_{1}\right) e^{i \delta_{22}}
\end{array}\right) .
$$

${ }^{4} \mathrm{~A}$ more general treatment could allow the possible dependence to complex phases

$$
\left|m_{i j}\right|=\left|m_{i j}\right|\left(m_{1}, m_{2}, \delta_{11}, \delta_{12}, \delta_{21}, \delta_{22}\right) .
$$

However, we will assume from this point onwards that the moduli of the matrix elements do not have this dependence. 


\subsection{Implications of $m_{11}=0$}

We define the singular value decomposition of the mass matrix as

$$
\mathbf{m}=\mathbf{L}^{\dagger} \mathbf{\Sigma R}
$$

where $\boldsymbol{\Sigma}=\operatorname{diag}\left(m_{1}, m_{2}\right)$ and $\mathbf{L}$ and $\mathbf{R}$ are the unitary transformations which bring the left and right Hermitian products, $\mathbf{m m}^{\dagger}$ and $\mathbf{m}^{\dagger} \mathbf{m}$, to diagonal form. The latter equation can be written explicitly as

$$
\mathbf{m}=m_{1}\left(\begin{array}{ll}
L_{11}^{*} R_{11} & L_{11}^{*} R_{12} \\
L_{12}^{*} R_{11} & L_{12}^{*} R_{12}
\end{array}\right)+m_{2}\left(\begin{array}{ll}
L_{21}^{*} R_{21} & L_{21}^{*} R_{22} \\
L_{22}^{*} R_{21} & L_{22}^{*} R_{22}
\end{array}\right)
$$

which plainly says to us that in general $m_{11}$ is different from zero,

$$
m_{11}=m_{1} L_{11}^{*} R_{11}+m_{2} L_{21}^{*} R_{21} \neq 0 .
$$

Considering the matrices $\mathbf{L}$ and $\mathbf{R}$ as $S U(2)$ transformations with their rotation angle satisfying $\tan ^{2} \theta=m_{1} / m_{2}$ the latter equation transforms into

$$
m_{11}=\frac{m_{1} m_{2} \pm m_{1} m_{2} e^{-i\left(\delta_{L}-\delta_{R}\right)}}{m_{1}+m_{2}}
$$

where the choice in sign is the freedom of having one rotation clockwise and the other either the same or counter-clockwise, respectively. Therefore, if by means of the left and right unitary transformations we want to guarantee this relation to be zero then we should have $\delta_{L}=\delta_{R}$ and opposite rotations for the left- and right-handed fields or likewise $\delta_{L}=\delta_{R}+\pi$ with both rotations in the same direction.

Our final mass matrix becomes with $\delta \equiv \delta_{L}$

$$
\mathbf{m}=\left(\begin{array}{cc}
0 & \sqrt{m_{1} m_{2}} e^{-i \delta} \\
-\sqrt{m_{1} m_{2}} e^{i \delta} & m_{2}-m_{1}
\end{array}\right),
$$

which is an anti-Hermitian matrix with $\delta_{22}=0$ as implied by $\delta_{L}=\delta_{R}+\pi$.

In the three family case, it has been shown [30,31] that it suffices to take either real or purely imaginary mass matrix elements to reproduce the experimentally observed masses and mixings. We assume this same feature in the two family case. Hence, our phase will be constrained to take only the values $\delta=0, \pi / 2, \pi$, or $3 \pi / 2$. In fact, the same set of values can be inferred by recalling that in the two (three) family case a mixing parametrization requires one (four) independent parameter (parameters) to be fully described. It is only when the number of fermion families is $n \leq 3$ that a mixing parametrization has enough fermion mass ratios to contain the number of mixing parameters [3]. And thus, as the two (four) mass ratios parametrization indicates, phases should not play any role except 
to determine if a rotation is real or its off-diagonal elements are purely imaginary with a freedom of choosing it either clockwise or anti-clockwise [3].

Now, inquiring the nature of such Yukawa interactions one is led to think about a possible origin for such a matrix form. A look into its hierarchical structure suggests a natural separation among its elements

$$
\mathbf{m}=\left(m_{2}-m_{1}\right)\left(\begin{array}{ll}
0 & 0 \\
0 & 1
\end{array}\right)+\sqrt{m_{1} m_{2}}\left(\begin{array}{cc}
0 & e^{-i \delta} \\
-e^{i \delta} & 0
\end{array}\right) .
$$

Note that the first term is a symmetric matrix and when $\delta=0$ or $\pi$ the second term is antisymmetric whereas symmetric for $\delta=\pi / 2$ or $3 \pi / 2$. Are these matrix structures dictated by some principle?

\section{A symmetrical origin}

Why are Yukawa interactions not flavor-blind though gauge interactions are? With this we mean that if $n$ fields had the same gauge interactions and gauge couplings, then the strength of their Yukawa couplings to the Higgs field should not distinguish the flavor. The most general form for this assertion is to introduce Yukawa couplings $\mathbf{y}_{i j}$ which are invariant under permutations of the massless families, that is flavor-blind Yukawa couplings (see Appendix $(\mathrm{B})$.

\subsection{Two fermion families}

Two fermion families coupling without distinction to the Higgs field imply

$$
\mathbf{y}^{1 \leftrightarrow 2}=y\left(\begin{array}{ll}
1 & 1 \\
1 & 1
\end{array}\right)
$$

This basis in commonly known as the democratic basis. Then in return, after the weak basis transformation

$$
O_{2}=\frac{1}{\sqrt{2}}\left(\begin{array}{cc}
1 & 1 \\
-1 & 1
\end{array}\right)
$$

the latter Yukawa couplings imply one massless, $m_{1}=0$, and one massive family, $m_{2}=2 y v$ with $v \equiv\langle H\rangle$ the nonvanishing vacuum expectation value of the neutral component of the Higgs field. This new basis is called the heavy o hierarchical basis. Here the Yukawa matrix takes the form

$$
\widetilde{\mathbf{y}}^{1 \leftrightarrow 2}=2 y\left(\begin{array}{ll}
0 & 0 \\
0 & 1
\end{array}\right) \text {. }
$$


The matrix in Eq. (31) has the symmetry properties of the group $S_{2 L} \otimes S_{2 R}$, that is invariance under the separate permutations of the rows and columns. At this point, it is not clear how the first family could acquire its mass. In fact, we have two choices: in the democratic basis we can either break $S_{2 L} \otimes S_{2 R}$ to $S_{2 S}$ or $S_{2 A}$, that is the symmetric or antisymmetric diagonal subgroup, respectively. The former is discarded as it will only give mass to the first family without inducing any mixing phenomena. On the other hand, the latter, described by the matrix term

$$
\mathbf{y}^{A}=\left(\begin{array}{cc}
\beta & \alpha \\
-\alpha & -\beta
\end{array}\right)
$$

will imply, after the weak basis transformation of Eq. (32),

$$
\widetilde{\mathbf{y}}^{A}=\left(\begin{array}{cc}
0 & \beta+\alpha \\
\beta-\alpha & 0
\end{array}\right) .
$$

In order to have the off-diagonal elements equal in magnitude, as in Eq. (30), we need $\alpha$ to be purely imaginary. Thus,

$$
\widetilde{\mathbf{y}}^{A}=\left(\begin{array}{cc}
0 & |y|_{A} e^{-i \delta_{A}} \\
|y|_{A} e^{i \delta_{A}} & 0
\end{array}\right)
$$

where $|y|_{A}^{2}=\alpha^{2}+\beta^{2}$ and $\tan \delta_{A}=-\alpha / \beta$. When $\delta_{A}=0$ or $\pi(\alpha=0)$ our matrix is symmetric and when $\delta_{A}=\pi / 2$ or $3 \pi / 2(\beta=0)$ then our matrix becomes antisymmetric. Just what we were looking for. The need for the symmetry breaking pattern $S_{2 L} \otimes S_{2 R} \rightarrow S_{2 A}$ in the two family case was recognized before [2], although it was found under the ansatz of an Hermitian mass matrix and the consideration that in some special basis one should have $y_{11}=0$.

Hence in the two family case, we have found a statement equivalent to our unique condition for reproducing the GST relation: Yukawa couplings shall be either flavor-blind or decomposed into several sets obeying distinct permutation symmetries. We shall call this statement the flavor-blind principle. In this sense, we are taking the concept of flavor-blind gauge interactions to the Yukawa sector by considering Yukawa couplings which cannot distinguish massless fields with equal quantum numbers.

\subsection{Three fermion families}

A recent study of fermion mixing in terms of mass ratios [3] showed that the three by three complex mass matrix has a hierarchical structure given by

$$
\mathcal{M} \sim f_{3}\left(\begin{array}{ccc}
0 & 0 & 0 \\
0 & 0 & 0 \\
0 & 0 & 1
\end{array}\right)+f_{2}\left(\begin{array}{ccc}
0 & 0 & 0 \\
0 & 0 & \times \\
0 & \times & 0
\end{array}\right)+f_{1}\left(\begin{array}{ccc}
0 & \times & \times \\
\times & 0 & 0 \\
\times & 0 & 0
\end{array}\right)
$$


with $f_{i}=f_{i}\left(m_{1}, m_{2}, m_{3}\right)$ an homogeneous function of degree one in $m_{3}$ and obeying the relation $f_{3} \gg f_{2} \gg f_{1}$. The particular feature of [3] is that the three fermion families mixing is decomposed in the study of successive two fermion families mixing. In fact, two independent questions emerge and should concern us: first, what lies behind the hierarchical nature between the matrix terms? and second, how these different Yukawa matrices appear? This work gives an explanation to the latter inquiry.

We wish now to apply our flavor-blind principle to the three family case to find out how it works in this scenario. Similarly, as in the two family case, the first term originates from the fact that three undistinguishable fermion families are coupling to the Higgs field

$$
\mathcal{Y}^{1 \leftrightarrow 2 \leftrightarrow 3}=y\left(\begin{array}{ccc}
1 & 1 & 1 \\
1 & 1 & 1 \\
1 & 1 & 1
\end{array}\right)
$$

The symmetry property of this matrix is $S_{3 L} \otimes S_{3 R}$. After the weak basis transformation

$$
O_{3}=\left(\begin{array}{ccc}
\frac{1}{\sqrt{2}} & \frac{1}{\sqrt{6}} & \frac{1}{\sqrt{3}} \\
-\frac{1}{\sqrt{2}} & \frac{1}{\sqrt{6}} & \frac{1}{\sqrt{3}} \\
0 & -\frac{2}{\sqrt{6}} & \frac{1}{\sqrt{3}}
\end{array}\right)
$$

only one family becomes massive, $m_{3}=3 y v$, while the other two remain massless.

In the mass basis 5 we have two massless fermion families. Therefore, the next set of Yukawa couplings after returning to the initial democratic basis should be invariant under the permutations of the two massless families

$$
\mathcal{Y}^{1 \leftrightarrow 2}=\left(\begin{array}{ccc}
\beta & \beta & \alpha_{1} \\
\beta & \beta & \alpha_{1} \\
\alpha_{2} & \alpha_{2} & \gamma
\end{array}\right)
$$

where the symmetry property of this matrix is $S_{2 L} \otimes S_{2 R}$. Using the weak basis transformation of Eq. (39) this Yukawa matrix transforms into

$$
\widetilde{\mathcal{Y}}^{1 \leftrightarrow 2}=\left(\begin{array}{ccc}
0 & 0 & 0 \\
0 & -\frac{2}{3}\left(\alpha_{1}+\alpha_{2}-\beta-\gamma\right) & \frac{1}{3} \sqrt{2}\left(\alpha_{1}-2 \alpha_{2}+2 \beta-\gamma\right) \\
0 & \frac{1}{3} \sqrt{2}\left(-2 \alpha_{1}+\alpha_{2}+2 \beta-\gamma\right) & \frac{1}{3}\left(2\left(\alpha_{1}+\alpha_{2}+2 \beta\right)+\gamma\right)
\end{array}\right)
$$

Demanding it to reproduce the two family case, that is the structure appearing in Eq. (30) implies the set of solutions shown in Table 1

\footnotetext{
${ }^{5}$ Here the mass basis coincides with the heavy basis.
} 
Table 1: Application of the flavor-blind principle, in the three family case with two massive families, gets parametrized by the Yukawa couplings $\alpha_{1}, \alpha_{2}, \beta$, and $\gamma$. The requirement of reproducing the GST relation, as in the two family case, for each possible value of $\delta=0, \pi / 2, \pi$, or $3 \pi / 2$, implies a different solution, as shown below.

\begin{tabular}{c|cccc}
\hline \hline$\delta$ & $\alpha_{1}$ & $\alpha_{2}$ & $\beta$ & $\gamma$ \\
\hline \hline 0 & $-\frac{m_{2}}{3 v}+\frac{\sqrt{m_{2} m_{3}}}{\sqrt{2} v}$ & $-\frac{m_{2}}{3 v}-\frac{\sqrt{m_{2} m_{3}}}{\sqrt{2} v}$ & $-\frac{m_{2}}{3 v}$ & $-\frac{m_{2}}{3 v}$ \\
$\pi / 2$ & $-\frac{m_{2}}{3 v}+i \frac{\sqrt{m_{2} m_{3}}}{3 \sqrt{2} v}$ & $-\frac{m_{2}}{3 v}+i \frac{\sqrt{m_{2} m_{3}}}{3 \sqrt{2} v}$ & $-\frac{m_{2}}{3 v}-\frac{i}{3 v} \sqrt{2 m_{2} m_{3}}$ & $-\frac{m_{2}}{3 v}+\frac{2 i}{3 v} \sqrt{2 m_{2} m_{3}}$ \\
$\pi$ & $-\frac{m_{2}}{3 v}-\frac{\sqrt{m_{2} m_{3}}}{\sqrt{2} v}$ & $-\frac{m_{2}}{3 v}+\frac{\sqrt{m_{2} m_{3}}}{\sqrt{2} v}$ & $-\frac{m_{2}}{3 v}$ & $-\frac{m_{2}}{3 v}$ \\
$3 \pi / 2$ & $-\frac{m_{2}}{3 v}-i \frac{\sqrt{m_{2} m_{3}}}{3 \sqrt{2} v}$ & $-\frac{m_{2}}{3 v}-i \frac{\sqrt{m_{2} m_{3}}}{3 \sqrt{2} v}$ & $-\frac{m_{2}}{3 v}+\frac{i}{3 v} \sqrt{2 m_{2} m_{3}}$ & $-\frac{m_{2}}{3 v}-\frac{2 i}{3 v} \sqrt{2 m_{2} m_{3}}$ \\
\hline
\end{tabular}

We have reached the point where we have one massless family left. The following term brings the Yukawa couplings from $S_{2 L} \otimes S_{2 R}$ to its diagonal subgroup $S_{2 A}$ (see Appendix B for more details on why $S_{2 A}$ and not its symmetric counterpart, $S_{2 S}$ ):

$$
\mathcal{Y}^{A}=\left(\begin{array}{ccc}
\tau & i \mu & \nu_{1} \\
-i \mu & -\tau & -\nu_{1} \\
\nu_{2} & -\nu_{2} & 0
\end{array}\right)
$$

which after the weak basis transformation of Eq. (39) turns into

$$
\tilde{\mathcal{Y}}^{A}=\left(\begin{array}{ccc}
0 & \frac{i \mu-2 \nu_{1}+\tau}{\sqrt{3}} & \sqrt{\frac{2}{3}}\left(i \mu+\nu_{1}+\tau\right) \\
\frac{-i \mu-2 \nu_{2}+\tau}{\sqrt{3}} & 0 & 0 \\
\sqrt{\frac{2}{3}}\left(-i \mu+\nu_{2}+\tau\right) & 0 & 0
\end{array}\right)
$$

Here we need to stress the following. The solutions appearing in Table 1 correspond to two massive families. After the inclusion of Eq. (42) the solutions will suffer the following change

$$
m_{2} \rightarrow \frac{\Delta_{23}^{2}}{m_{3}}
$$

where $\Delta_{23}=\Delta_{23}\left(m_{1}, m_{2}, m_{3}\right)$ represents a sum of terms to be rotated away; giving in the end, the total needed rotation in the 2-3 sector of the corresponding fermion species $(f=u, d, e, \nu)$ [3].

The arbitrary parameters appearing in Eq. (42) can be solved in terms of $\Delta_{13}$ and $\Delta_{12}$. We can have four different cases: symmetry or antisymmetry in both the 1-2 and 1-3 sectors, or the two independent combinations. The four solutions are shown in Table 2. It is not our purpose to give the explicit expressions in terms of the masses, but rather to show how each new matrix term can be solved in a unique way. 
Table 2: Application of the flavor-blind principle, in the three family case with three massive families, gets parametrized by the Yukawa couplings $\tau, \mu, \nu_{1}$, and $\nu_{2}$. The requirement of reproducing the GST relation, as in the two family case, for each possible value of $\delta=0, \pi / 2, \pi$, or $3 \pi / 2$, implies a different solution, as shown below. In the left column we have denoted $\mathrm{S}$ and $\mathrm{A}$ to the two possibilities of having the off diagonal terms as either symmetric or antisymmetric, respectively.

\begin{tabular}{c|cccc}
\hline \hline (1-2)-(1-3) & $\tau$ & $\mu$ & $\nu_{1}$ & $\nu_{2}$ \\
\hline \hline S-S & $\frac{1}{3}\left(\sqrt{3} \Delta_{12}+\sqrt{6} \Delta_{13}\right)$ & 0 & $\frac{1}{\sqrt{6}}\left(-\sqrt{2} \Delta_{12}+\Delta_{13}\right)$ & $-\frac{1}{\sqrt{6}}\left(\sqrt{2} \Delta_{12}-\Delta_{13}\right)$ \\
A-A & 0 & $-\frac{i}{3}\left(\sqrt{3} \Delta_{12}+\sqrt{6} \Delta_{13}\right)$ & $\frac{1}{\sqrt{6}}\left(-\sqrt{2} \Delta_{12}+\Delta_{13}\right)$ & $\frac{1}{\sqrt{6}}\left(\sqrt{2} \Delta_{12}-\Delta_{13}\right)$ \\
S-A & $\frac{1}{\sqrt{3}} \Delta_{12}$ & $-i \sqrt{\frac{2}{3}} \Delta_{13}$ & $\frac{1}{\sqrt{6}}\left(-\sqrt{2} \Delta_{12}+\Delta_{13}\right)$ & $-\frac{1}{\sqrt{6}}\left(\sqrt{2} \Delta_{12}+\Delta_{13}\right)$ \\
A-S & $\sqrt{\frac{2}{3}} \Delta_{13}$ & $-i \frac{1}{\sqrt{3}} \Delta_{12}$ & $\frac{1}{\sqrt{6}}\left(-\sqrt{2} \Delta_{12}+\Delta_{13}\right)$ & $\frac{1}{\sqrt{6}}\left(\sqrt{2} \Delta_{12}+\Delta_{13}\right)$ \\
\hline
\end{tabular}

Let us summarize. In the three family case, the entirely Yukawa matrix for any fermion species is built as the sum of three different contributions

$$
\mathcal{Y}=\mathcal{Y}^{1 \leftrightarrow 2 \leftrightarrow 3}+\mathcal{Y}^{1 \leftrightarrow 2}+\mathcal{Y}^{A} .
$$

Each of them correspond to a step where a given number of fields were massless. Due to this and by the application of the flavor-blind principle, each matrix have been demanded to satisfy symmetry properties under permutations of the corresponding massless fields. Only in the last step, with a single massless family, the symmetry group is chosen as to produce mixing phenomena.

\section{Conclusions}

We have found, in the two family case, the minimal form the Yukawa matrices should satisfy in order to lead exactly to a Gatto-Sartori-Tonin (GST) like fomula for an individual fermion species, $\tan ^{2} \theta_{i j}^{f}=m_{f, i} / m_{f, j}(f=u, d, e, \nu)$. Furthermore, we have also found that this form can be obtained from demanding the Yukawa matrices to possess symmetry properties under certain permutations of its rows and/or columns. This symmetrical origin has been expressed by the following statement which we have called the flavor-blind principle: Yukawa couplings shall be either flavor-blind or decomposed into several sets obeying distinct permutation symmetries. Fermion mixing and the hierarchical massive nature of fermions can be studied as two independent features (see for example [16]): on one hand, some dynamics giving hierarchical masses and on the other, some mechanism producing the mixing in a given ordered way. The flavor-blind principle, in this respect, helps us to understand how mixing can be produced in such sequential manner. This condition can be applied to the general case of $n$ fermion families where the symmetry properties of the introduced set of Yukawa couplings 
follow a unique sequential breaking $S_{n L} \otimes S_{n R} \rightarrow S_{(n-1) L} \otimes S_{(n-1) R} \rightarrow \cdots \rightarrow S_{2 L} \otimes S_{2 R} \rightarrow S_{2 A}$. The particular cases of two and three families have been treated. We need to remark that both of them were already studied by other authors but under different considerations. Regarding our work, and its main difference with respect to previous ones, we have shown that the last Yukawa matrix that gives mass to the first family should always obey the symmetry properties of the diagonal and antisymmetric subgroup $S_{2 A}$. For last, the flavor-blind principle has helped us to understand why the four mass ratios parametrization found for the lepton sector [3] equally applies, as in the quark sector, in spite of the very mild hierarchy in the neutrino masses.

\section{Acknowledgements}

UJSS wants to thank U. Nierste for suggesting the approach of taking the Gatto-Sartori-Tonin relation as a starting point and studying thereof its consequences. Also the author wants to thank A. Aranda, O. G. Miranda, E. Peinado, and A. Vicente for a careful reading of the manuscript and their useful comments on it. After submission to the journal the author received many insights and comments from W. G. Hollik; he feels indebted to him. This work has been supported by the mexican grants CONACyT-166639 and SNI-Mexico.

\section{A The Gatto-Sartori-Tonin relation in a system with two coupled quantum harmonic oscillators}

The most general Hamiltonian describing two coupled quantum harmonic oscillators with different frequencies is

$$
\hat{H}=\frac{\hat{p}_{1}^{2}}{2 m_{1}}+\frac{\hat{p}_{2}^{2}}{2 m_{2}}+\frac{1}{2} k_{1} \hat{x}_{1}^{2}+\frac{1}{2} k_{2} \hat{x}_{2}^{2}+\frac{1}{2} k_{0}\left(\hat{x}_{2}-\hat{x}_{1}\right)^{2}
$$

where the $k_{i}$ are real parameters and $\hat{x}_{i}$ measures the displacement from the equilibrium point. Now, in order to decouple them, we first need to make a change of scale but which preserves the corresponding commuting relations

$$
\left[\hat{x}_{i}, \hat{p}_{j}\right]=i \hbar \delta_{i j}, \quad\left[\hat{x}_{i}, \hat{x}_{j}\right]=0, \quad\left[\hat{p}_{i}, \hat{p}_{j}\right]=0
$$

This change of scale is found to be given by

$$
\hat{p}_{1}^{2}=\sqrt{\frac{m_{1}}{m_{2}}} \hat{P}_{1}^{2}, \quad \hat{x}_{1}^{2}=\sqrt{\frac{m_{2}}{m_{1}}} \hat{X}_{1}^{2}, \quad \hat{p}_{2}^{2}=\sqrt{\frac{m_{2}}{m_{1}}} \hat{P}_{2}^{2}, \quad \hat{x}_{2}^{2}=\sqrt{\frac{m_{1}}{m_{2}}} \hat{X}_{2}^{2} .
$$


The new Hamiltonian becomes

$$
\hat{H}=\frac{\hat{P}_{1}^{2}}{2 \mu}+\frac{\hat{P}_{2}^{2}}{2 \mu}+\frac{1}{2} \mu w_{1}^{2} \hat{X}_{1}^{2}+\frac{1}{2} \mu w_{2}^{2} \hat{X}_{2}^{2}+\frac{1}{2} k_{0}\left(\sqrt{\frac{m_{2}}{m_{1}}} \hat{X}_{1}^{2}+\sqrt{\frac{m_{1}}{m_{2}}} \hat{X}_{2}^{2}+2 \hat{X}_{1} \hat{X}_{2}\right),
$$

where $\mu=\sqrt{m_{1} m_{2}}$ is the geometric mass. The system can be brought to diagonal form by means of an orthogonal transformation

$$
\left(\begin{array}{c}
\hat{X}_{+} \\
\hat{X}_{-}
\end{array}\right)=\left(\begin{array}{c}
\cos \theta \hat{X}_{1}-\sin \theta \hat{X}_{2} \\
\sin \theta \hat{X}_{1}+\cos \theta \hat{X}_{2}
\end{array}\right)
$$

with the angle of rotation satisfying

$$
\tan 2 \theta=\frac{-2 k_{0}}{\mu\left(w_{2}^{2}-w_{1}^{2}\right)+k_{0} \frac{m_{1}-m_{2}}{\mu}}
$$

Thus, the Hamiltonian in terms of the two normal modes now describes two uncoupled harmonic oscillators with equal masses

$$
\hat{H}=\frac{\hat{P}_{+}^{2}}{2 \mu}+\frac{\hat{P}_{-}^{2}}{2 \mu}+\frac{1}{2} \mu w_{+}^{2} \hat{X}_{+}^{2}+\frac{1}{2} \mu w_{-}^{2} \hat{X}_{-}^{2},
$$

but two different frequencies

$$
\begin{aligned}
& w_{+}^{2}=w_{1}^{2} \sin ^{2} \theta+w_{2}^{2} \cos ^{2} \theta+\frac{k_{0}}{\mu}\left(\sqrt[4]{\frac{m_{2}}{m_{1}}} \cos \theta+\sqrt[4]{\frac{m_{1}}{m_{2}}} \sin \theta\right)^{2} \\
& w_{-}^{2}=w_{1}^{2} \cos ^{2} \theta+w_{2}^{2} \sin ^{2} \theta+\frac{k_{0}}{\mu}\left(\sqrt[4]{\frac{m_{1}}{m_{2}}} \cos \theta-\sqrt[4]{\frac{m_{2}}{m_{1}}} \sin \theta\right)^{2} .
\end{aligned}
$$

If we ask the system to be in resonance then the initial frequencies need to be equal, $w=w_{1}=w_{2}$. This simplification with physical meaning implies that the rotation angle will satisfy the quadratic equation

$$
\tan ^{2} \theta+\frac{m_{2}-m_{1}}{\mu} \tan \theta-1=0
$$

that is

$$
\tan \theta=\left\{\begin{array}{l}
-\sqrt{\frac{m_{2}}{m_{1}}} \\
+\sqrt{\frac{m_{1}}{m_{2}}}
\end{array},\right.
$$


and the new normal frequencies will be

$$
\begin{aligned}
& w_{+}^{2}=w^{2}+\frac{k_{0}}{\mu}\left(\sqrt[4]{\frac{m_{2}}{m_{1}}} \cos \theta-\sqrt[4]{\frac{m_{1}}{m_{2}}} \sin \theta\right)^{2}=\left\{\begin{array}{l}
w^{2} \\
w^{2}+\frac{k_{0}}{\mu^{2}}\left(m_{1}+m_{2}\right),
\end{array}\right. \\
& w_{-}^{2}=w^{2}+\frac{k_{0}}{\mu}\left(\sqrt[4]{\frac{m_{1}}{m_{2}}} \cos \theta+\sqrt[4]{\frac{m_{2}}{m_{1}}} \sin \theta\right)^{2}=\left\{\begin{array}{l}
w^{2}+\frac{k_{0}}{\mu^{2}}\left(m_{1}+m_{2}\right) \\
w^{2}
\end{array}\right.
\end{aligned}
$$

Both types of solutions give the same kind of normal frequencies. What we found out from this exercise is that the angle of rotation, which brings our system to its normal modes, satisfy the GST relation

$$
\tan ^{2} \theta=\frac{m_{1}}{m_{2}}
$$

Hence the GST relation originates, in this case, by uncoupling and demanding resonance (same frequencies) to the two coupled quantum harmonic oscillators with different masses.

\section{B Application of the flavor-blind principle to the $n$ family case}

Let us consider $n$ fermion families, that is $n$ fermion fields with universal gauge couplings. Their kinetic terms when putted together span an $n$-dimensional family space. Any unitary transformation acting in the $n$ family vector of each gauge representation will leave the kinetic terms invariant. This group commonly known as the flavor group is given as 6

$$
\mathcal{G}_{F}=U(n)_{L}^{Q} \otimes U(n)_{R}^{u} \otimes U(n)_{R}^{d} \otimes U(n)_{L}^{\ell} \otimes U(n)_{R}^{e} \otimes U(n)_{R}^{\nu}
$$

Application of the flavor-blind principle tells us how to introduce the Yukawa couplings. For a generic fermion species the first matrix term cannot distinguish among the $n$ fermion fields. Thus,

$$
\mathcal{Y}^{1 \leftrightarrow 2 \leftrightarrow 3 \leftrightarrow \cdots \leftrightarrow(n-1) \leftrightarrow n}=y_{n}\left(\begin{array}{ccccc}
1 & 1 & 1 & \cdots & 1 \\
1 & 1 & 1 & \cdots & 1 \\
1 & 1 & 1 & \cdots & 1 \\
\vdots & \vdots & \vdots & \vdots & \vdots \\
1 & 1 & 1 & \cdots & 1
\end{array}\right) \text {. }
$$

\footnotetext{
${ }^{6}$ For the sake of clarity we choose to work only with the Dirac case.
} 
This basis is known as the democratic basis. The symmetry properties of this matrix are described by the group $S_{n L} \otimes S_{n R}$. Usage of the weak basis transformation

$$
O_{n}=\left(\begin{array}{cccccc}
\frac{1}{\sqrt{2}} & \frac{1}{\sqrt{3 \cdot 2}} & \frac{1}{\sqrt{4 \cdot 3}} & \cdots & \frac{1}{\sqrt{n(n-1)}} & \frac{1}{\sqrt{n}} \\
-\frac{1}{\sqrt{2}} & \frac{1}{\sqrt{3 \cdot 2}} & \frac{1}{\sqrt{4 \cdot 3}} & \cdots & \frac{1}{\sqrt{n(n-1)}} & \frac{1}{\sqrt{n}} \\
0 & -\frac{2}{\sqrt{3 \cdot 2}} & \frac{1}{\sqrt{4 \cdot 3}} & \cdots & \frac{1}{\sqrt{n(n-1)}} & \frac{1}{\sqrt{n}} \\
0 & 0 & -\frac{3}{\sqrt{4 \cdot 3}} & \cdots & \frac{1}{\sqrt{n(n-1)}} & \frac{1}{\sqrt{n}} \\
\vdots & \vdots & \vdots & \cdots & \vdots & \vdots \\
0 & 0 & 0 & \cdots & \frac{1}{\sqrt{n(n-1)}} & \frac{1}{\sqrt{n}} \\
0 & 0 & 0 & \cdots & -\frac{n-1}{\sqrt{n(n-1)}} & \frac{1}{\sqrt{n}}
\end{array}\right),
$$

brings all Yukawa matrices to the form

$$
\widetilde{\mathcal{Y}}^{1 \leftrightarrow 2 \leftrightarrow 3 \leftrightarrow \cdots \leftrightarrow(n-1) \leftrightarrow n}=n y_{n}\left(\begin{array}{ccccc}
0 & 0 & 0 & \cdots & 0 \\
0 & 0 & 0 & \cdots & 0 \\
0 & 0 & 0 & \cdots & 0 \\
\vdots & \vdots & \vdots & \vdots & \vdots \\
0 & 0 & 0 & \cdots & 1
\end{array}\right) .
$$

From Eq. (61) we already know we will have a single massive family, as it is a rank one matrix. In the new basis, or heavy basis, the mass term is $m_{n}=n y_{n} v$ with $v \equiv\langle H\rangle$ the nonvanishing vacuum expectation value of the neutral component of the Higgs field. Meanwhile, $\mathcal{G}_{F}$ has broken to $U(n-1)^{6}$.

Again, the flavor-blind principle helps us to introduce the new Yukawa couplings. In the democratic basis we have

$$
\mathcal{Y}^{1 \leftrightarrow 2 \leftrightarrow 3 \leftrightarrow \cdots \leftrightarrow(n-1)}=\left(\begin{array}{ccccc}
\beta_{n-1} & \beta_{n-1} & \cdots & \beta_{n-1} & \alpha_{n-1} \\
\beta_{n-1} & \beta_{n-1} & \cdots & \beta_{n-1} & \alpha_{n-1} \\
\vdots & \vdots & \cdots & \vdots & \vdots \\
\beta_{n-1} & \beta_{n-1} & \cdots & \beta_{n-1} & \alpha_{n-1} \\
\alpha_{n-1}^{\prime} & \alpha_{n-1}^{\prime} & \cdots & \alpha_{n-1}^{\prime} & \gamma_{n-1}
\end{array}\right) .
$$

This is a rank two matrix. The arbitrary parameters $\alpha_{n-1}, \beta_{n-1}, \alpha_{n-1}^{\prime}$, and $\gamma_{n-1}$ are in general complex parameters; all of them depending in $m_{n-1} / v$ and $m_{n} / v$. In the latter case as an homogeneous function of first order. Whenever $m_{n-1}=0$ all of them are also equal to zero. The $(n-1)$ dimensional submatrix in the upper left corner suggests the possibility of extracting a similar matrix 
as in Eq. (61). This is only telling us that, after the weak basis transformation of Eq. (62), the $(n, n)$ element in Eq. (63) will gain a contribution proportional to $m_{n-1}$. The heavy basis shows explicitly how $U(n-1)^{6}$ has broken to $U(n-2)^{6}$ with only four non-zero matrix elements

$$
\begin{array}{r}
\widetilde{\mathcal{Y}}_{n-1, n-1}^{1 \leftrightarrow 2 \leftrightarrow 3 \leftrightarrow \cdots \leftrightarrow(n-1)}=\frac{n-1}{n}\left[\beta_{n-1}+\gamma_{n-1}-\alpha_{n-1}-\alpha_{n-1}^{\prime}\right], \\
\widetilde{\mathcal{Y}}_{n-1, n}^{1 \leftrightarrow 2 \leftrightarrow 3 \leftrightarrow \cdots \leftrightarrow(n-1)}=\frac{\sqrt{n-1}}{n}\left[(n-1)\left(\beta_{n-1}-\alpha_{n-1}^{\prime}\right)+\alpha_{n-1}-\gamma_{n-1}\right], \\
\widetilde{\mathcal{Y}}_{n, n-1}^{1 \leftrightarrow 2 \leftrightarrow 3 \leftrightarrow \cdots \leftrightarrow(n-1)}=\frac{\sqrt{n-1}}{n}\left[(n-1)\left(\beta_{n-1}-\alpha_{n-1}\right)+\alpha_{n-1}^{\prime}-\gamma_{n-1}\right], \\
\widetilde{\mathcal{Y}}_{n, n}^{1 \leftrightarrow 2 \leftrightarrow 3 \leftrightarrow \cdots \leftrightarrow(n-1)}=\frac{1}{n}\left\{\gamma_{n-1}+(n-1)\left[(n-1) \beta_{n-1}+\alpha_{n-1}+\alpha_{n-1}^{\prime}\right]\right\} .
\end{array}
$$

That is, $n-2$ families are massless. The flavor-blind principle tells us that the new Yukawa couplings to be introduced in the democratic basis should be

$$
\mathcal{Y}^{1 \leftrightarrow 2 \leftrightarrow 3 \leftrightarrow \cdots \leftrightarrow(n-2)}=\left(\begin{array}{cccccc}
\beta_{n-2} & \beta_{n-2} & \cdots & \beta_{n-2} & \omega_{n-2} & \alpha_{n-2} \\
\beta_{n-2} & \beta_{n-2} & \cdots & \beta_{n-2} & \omega_{n-2} & \alpha_{n-2} \\
\vdots & \vdots & \cdots & \vdots & \vdots & \vdots \\
\beta_{n-2} & \beta_{n-2} & \cdots & \beta_{n-2} & \omega_{n-2} & \alpha_{n-2} \\
\omega_{n-2}^{\prime} & \omega_{n-2}^{\prime} & \cdots & \omega_{n-2}^{\prime} & \rho_{n-2} & \lambda_{n-2} \\
\alpha_{n-2}^{\prime} & \alpha_{n-2}^{\prime} & \cdots & \alpha_{n-2}^{\prime} & \lambda_{n-2}^{\prime} & \gamma_{n-2}
\end{array}\right) .
$$

which after the weak basis transformation the only non-zero elements are those conforming the $3 \times 3$ submatrix

$$
\begin{array}{r}
\widetilde{\mathcal{Y}}_{n-2, n-2}^{1 \leftrightarrow 2 \leftrightarrow 3 \leftrightarrow \cdots \leftrightarrow(n-2)}=\frac{n-2}{n-1}\left[\beta_{n-2}+\rho_{n-2}-\omega_{n-2}-\omega_{n-2}^{\prime}\right], \\
\widetilde{\mathcal{Y}}_{n-2, n-1}^{1 \leftrightarrow 2 \leftrightarrow 3 \leftrightarrow \cdots \leftrightarrow(n-2)}=\sqrt{\frac{n-2}{n-1}}\left[(n-2)\left(\beta_{n-2}-\omega_{n-2}^{\prime}\right)-(n-1)\left(\alpha_{n-2}-\lambda_{n-2}\right)+\omega_{n-2}-\rho_{n-2}\right], \\
\widetilde{\mathcal{Y}}_{n-2, n}^{1 \leftrightarrow 2 \leftrightarrow 3 \leftrightarrow \cdots \leftrightarrow(n-2)}=\sqrt{\frac{n-2}{n(n-1)}}\left[(n-2)\left(\beta_{n-2}-\omega_{n-2}^{\prime}\right)+\alpha_{n-2}-\lambda_{n-2}+\omega_{n-2}-\rho_{n-2}\right], \\
\widetilde{\mathcal{Y}}_{n-1, n-2}^{1 \leftrightarrow 2 \leftrightarrow 3 \leftrightarrow \cdots \leftrightarrow(n-2)}=\sqrt{\frac{n-2}{n-1}}\left[(n-2)\left(\beta_{n-2}-\omega_{n-2}\right)-(n-1)\left(\alpha_{n-2}^{\prime}-\lambda_{n-2}^{\prime}\right)+\omega_{n-2}^{\prime}-\rho_{n-2}\right], \\
\widetilde{\mathcal{Y}}_{n-1, n-1}^{1 \leftrightarrow 2 \leftrightarrow 3 \leftrightarrow \cdots \leftrightarrow(n-2)}=\frac{1}{n(n-1)}\left\{(n-2)\left[(n-2) \beta_{n-2}+\omega_{n-2}^{\prime}+\omega_{n-2}+\alpha_{n-2}+\alpha_{n-2}^{\prime}\right]+\right. \\
\left.(n-1)\left[(n-1) \gamma_{n-2}-\lambda_{n-2}^{\prime}-\lambda_{n-2}\right]+\rho_{n-2},\right\}
\end{array}
$$




$$
\begin{array}{r}
\widetilde{\mathcal{Y}}_{n-1, n}^{1 \leftrightarrow 2 \leftrightarrow 3 \leftrightarrow \cdots \leftrightarrow(n-2)}=\frac{1}{n \sqrt{n-1}}\left\{(n-2)\left[(n-2) \beta_{n-2}+\omega_{n-2}^{\prime}+\omega_{n-2}-(n-1) \alpha_{n-2}^{\prime}+\alpha_{n-2}\right]\right. \\
\left.-(n-1)\left(\gamma_{n-2}+\lambda_{n-2}^{\prime}\right)+\rho_{n-2}+\lambda_{n-2}\right\}, \\
\widetilde{\mathcal{Y}}_{n, n-2}^{1 \leftrightarrow 2 \leftrightarrow 3 \leftrightarrow \cdots \leftrightarrow(n-2)}=\sqrt{\frac{n-2}{n(n-1)}\left[(n-2)\left(\beta_{n-2}-\omega_{n-2}\right)+\alpha_{n-2}^{\prime}-\lambda_{n-2}^{\prime}+\omega_{n-2}^{\prime}-\rho_{n-2}\right],} \\
\widetilde{\mathcal{Y}}_{n, n-1}^{1 \leftrightarrow 2 \leftrightarrow 3 \leftrightarrow \cdots \leftrightarrow(n-2)}=\frac{1}{n \sqrt{n-1}}\left\{(n-2)\left[(n-2) \beta_{n-2}+\omega_{n-2}+\omega_{n-2}^{\prime}-(n-1) \alpha_{n-2}+\alpha_{n-2}^{\prime}\right]\right. \\
\left.-(n-1)\left(\gamma_{n-2}+\lambda_{n-2}\right)+\rho_{n-2}+\lambda_{n-2}^{\prime}\right\}, \\
\widetilde{\mathcal{Y}}_{n, n}^{1 \leftrightarrow 2 \leftrightarrow 3 \leftrightarrow \cdots \leftrightarrow(n-2)}=\frac{1}{n}\left\{(n-2)\left[(n-2) \beta_{n-2}+\omega_{n-2}^{\prime}+\omega_{n-2}+\alpha_{n-2}^{\prime}+\alpha_{n-2}\right]+\right. \\
\left.\rho_{n-2}+\gamma_{n-2}+\lambda_{n-2}^{\prime}+\lambda_{n-2}\right\} .
\end{array}
$$

Inclusion of further terms to give masses to the $n-2$ fermions left will follow the same logic as given by the flavor-blind principle.

For last, let us discuss why the last step of symmetry breaking is given by $S_{2 L} \otimes S_{2 R} \rightarrow S_{2 A}$ instead of $S_{2 L} \otimes S_{2 R} \rightarrow S_{2 S}$. In the former case, the Yukawa couplings are given by

$$
\mathcal{Y}^{A}=\left(\begin{array}{ccccc}
\beta_{1} & \xi_{1} & \lambda_{1} & \ldots & \alpha_{1} \\
-\xi_{1} & -\beta_{1} & -\lambda_{1} & \ldots & -\alpha_{1} \\
\varsigma_{1} & -\varsigma_{1} & 0 & \ldots & c_{1} \\
\vdots & \vdots & \vdots & \vdots & \vdots \\
\mu_{1} & -\mu_{1} & c_{1}^{\prime} & \cdots & 0
\end{array}\right)
$$

whereas for the latter

$$
\mathcal{Y}^{S}=\left(\begin{array}{ccccc}
\beta_{1} & \xi_{1} & \lambda_{1} & \cdots & \alpha_{1} \\
\xi_{1} & \beta_{1} & \lambda_{1} & \cdots & \alpha_{1} \\
\varsigma_{1} & \varsigma_{1} & a_{1} & \cdots & c_{1} \\
\vdots & \vdots & \vdots & \vdots & \vdots \\
\mu_{1} & \mu_{1} & c_{1}^{\prime} & \cdots & z_{1}
\end{array}\right)
$$

A straightforward calculation shows that after the corresponding electroweak basis transformation 
one gets the generic forms 7

$$
\widetilde{\mathcal{Y}}^{A} \sim\left(\begin{array}{ccccc}
0 & \times & \times & \cdots & \times \\
\times & 0 & \times & \cdots & \times \\
\times & \times & \times & \cdots & \times \\
\vdots & \vdots & \vdots & \vdots & \vdots \\
\times & \times & \times & \cdots & \times
\end{array}\right)
$$

and

$$
\widetilde{\mathcal{Y}}^{S} \sim\left(\begin{array}{ccccc}
\times & 0 & 0 & \cdots & 0 \\
0 & \times & \times & \cdots & \times \\
0 & \times & \times & \cdots & \times \\
\vdots & \vdots & \vdots & \vdots & \vdots \\
0 & \times & \times & \cdots & \times
\end{array}\right) .
$$

From this picture it becomes clear why $S_{2 L} \otimes S_{2 R} \rightarrow S_{2 A}$ is the correct symmetry breaking step as it is the only one which implies mixing between the first family with the other fermion families.

\section{References}

[1] R. Gatto, G. Sartori, and M. Tonin, "Weak Selfmasses, Cabibbo Angle, and Broken SU(2) x SU(2)," Phys.Lett. B28 (1968) 128-130.

[2] H. Lehmann, C. Newton, and T. T. Wu, "A New variant of symmetry breaking for quark mass matrices," Phys. Lett. B384 (1996) 249-254.

[3] W. G. Hollik and U. J. Saldaña Salazar, "The double mass hierarchy pattern: simultaneously understanding quark and lepton mixing," Nucl.Phys. B892 (2015) 364-389. arXiv:1411.3549 [hep-ph].

[4] H. Fritzsch and Z.-z. Xing, "Mass and flavor mixing schemes of quarks and leptons," Prog.Part.Nucl.Phys. 45 (2000) 1-81, arXiv: hep-ph/9912358 [hep-ph].

[5] H. Fritzsch, "Hierarchical Chiral Symmetries and the Quark Mass Matrix," Phys.Lett. B184 (1987) 391.

[6] L. J. Hall and A. Rasin, "On the generality of certain predictions for quark mixing," Phys.Lett. B315 (1993) 164-169, arXiv: hep-ph/9303303 [hep-ph].

\footnotetext{
${ }^{7}$ Only for $n=3$ the generic form of $\widetilde{\mathcal{Y}}^{A}$ in Eq. (81) acquires the form written in Eq. (43).
} 
[7] Z.-z. Xing, "Implications of the quark mass hierarchy on flavor mixings," J.Phys. G23 (1997) 1563-1578, arXiv: hep-ph/9609204 [hep-ph],

[8] A. Rasin, "Diagonalization of quark mass matrices and the Cabibbo-Kobayashi-Maskawa matrix," arXiv:hep-ph/9708216 [hep-ph].

[9] A. Rasin, "Hierarchical quark mass matrices," Phys.Rev. D58 (1998) 096012, arXiv: hep-ph/9802356 [hep-ph].

[10] J. Chkareuli and C. Froggatt, "Where does flavor mixing come from?," Phys.Lett. B450 (1999) 158-164, arXiv: hep-ph/9812499 [hep-ph].

[11] H. Fritzsch and Z.-z. Xing, "The Light quark sector, CP violation, and the unitarity triangle," Nucl.Phys. B556 (1999) 49-75, arXiv: hep-ph/9904286 [hep-ph].

[12] J. Chkareuli, C. Froggatt, and H. Nielsen, "Minimal mixing of quarks and leptons in the SU(3) theory of flavor," Nucl.Phys. B626 (2002) 307-343, arXiv:hep-ph/0109156 [hep-ph].

[13] S. Morisi, E. Peinado, Y. Shimizu, and J. W. F. Valle, "Relating quarks and leptons without grand-unification," Phys. Rev. D84 (2011) 036003, arXiv: 1104.1633 [hep-ph].

[14] S. Morisi and E. Peinado, "An A(4) model for lepton masses and mixings," Phys. Rev. D80 (2009) 113011, arXiv:0910.4389 [hep-ph].

[15] Z.-z. Xing, "Model-independent access to the structure of quark flavor mixing," Phys.Rev. D86 (2012) 113006, arXiv: 1211.3890 [hep-ph].

[16] S. Knapen and D. J. Robinson, "Disentangling Mass and Mixing Hierarchies," Phys. Rev. Lett. 115 no. 16, (2015) 161803, arXiv:1507.00009 [hep-ph],

[17] H. Harari, H. Haut, and J. Weyers, "Quark Masses and Cabibbo Angles," Phys. Lett. B78 (1978) 459.

[18] P. Kaus and S. Meshkov, “A BCS Quark Mass Matrix,” Mod.Phys.Lett. A3 (1988) 1251.

[19] L. Lavoura, "A Relationship Between the Democratic Family Mixing and the Fritzsch Schemes for the Mass Matrices," Phys.Lett. B228 (1989) 245.

[20] H. Fritzsch and J. Plankl, "Flavor Democracy and the Lepton - Quark Hierarchy," Phys. Lett. B237 (1990) 451. 
[21] M. Tanimoto, "New Quark Mass Matrix Based on the BCS Form," Phys. Rev. D41 (1990) 1586.

[22] P. Kaus and S. Meshkov, "Generational Mass Generation and Symmetry Breaking," Phys. Rev. D42 (1990) 1863-1867.

[23] K. S. Babu and R. N. Mohapatra, "Permutation Symmetry and the Origin of Fermion Mass Hierarchy," Phys. Rev. Lett. 64 (1990) 2747.

[24] H. Fritzsch and D. Holtmannspotter, "The Breaking of subnuclear democracy as the origin of flavor mixing," Phys. Lett. B338 (1994) 290-294, arXiv: hep-ph/9406241 [hep-ph].

[25] A. Mondragon and E. Rodriguez-Jauregui, "The Breaking of the flavor permutational symmetry: Mass textures and the CKM matrix," Phys.Rev. D59 (1999) 093009, arXiv:hep-ph/9807214 [hep-ph].

[26] J. Barranco, F. Gonzalez Canales, and A. Mondragon, "Universal Mass Texture, CP violation and Quark-Lepton Complementarity," Phys.Rev. D82 (2010) 073010, arXiv:1004.3781 [hep-ph],

[27] A. Ibarra and A. Solaguren-Beascoa, "Radiative Generation of Quark Masses and Mixing Angles in the Two Higgs Doublet Model," Phys. Lett. $\mathbf{B 7 3 6}$ (2014) 16-19, arXiv:1403.2382 [hep-ph].

[28] W. Altmannshofer, C. Frugiuele, and R. Harnik, "Fermion Hierarchy from Sfermion Anarchy," JHEP 12 (2014) 180, arXiv:1409.2522 [hep-ph].

[29] A. Ibarra and A. Solaguren-Beascoa, "Lepton parameters in the see-saw model extended by one extra Higgs doublet," JHEP 11 (2014) 089, arXiv:1409.5011 [hep-ph],

[30] I. Masina, "A Maximal atmospheric mixing from a maximal CP violating phase," Phys.Lett. B633 (2006) 134-140, arXiv: hep-ph/0508031 [hep-ph].

[31] I. Masina and C. A. Savoy, "Real and imaginary elements of fermion mass matrices," Nucl.Phys. B755 (2006) 1-20, arXiv: hep-ph/0603101 [hep-ph]. 